\title{
Aplastic anaemia associated with organochlorine pesticide: case reports and review of evidence
}

\author{
F P Rugman, R Cosstick
}

\begin{abstract}
Three patients with aplastic anaemia had a history of substantial previous exposure to organochlorine pesticides. The temporal association between chemical exposure and the onset of first symptoms of anaemia was strongly supportive. Organochlorines have the property of lipid affinity and accumulation in adipose tissue. Objective evidence of clinically important concentrations of tissue pesticide residues may be a useful confirmation of previous exposure. In the patients studied the presence of Lindane $(\gamma$ hexachlorocyclohexane) was shown using gas chromatography/mass spectrometry with selective ion monitoring of fragments obtained from one heavily exposed patient, with concentrations about five times greater than a matched control. The presence of clinically important tissue concentrations of pentachlorophenol was also confirmed in a second patient exposed to this agent.

The long term safety of organochlorine pesticides remains doubtful as they were introduced before adequate toxicological screening tests had been developed. The central registration of possible haematological adverse reactions, however, forms an important epidemiological method in the study of environmental chemical hazards and should be complied with whenever possible.
\end{abstract}

\section{Case reports}

CASE 1

A 12 year old boy presented with a four month history of pallor, easy bruising, exertional dyspnoea and headache. No antibiotics or other medication had been given. On admission he had noticeable pallor of skin and mucous membranes, with peripheral bruising. Height and weight were normal for age. Radial or thumb abnormalities, cutaneous hyperpigmentation, or neurological abnormalities were not present. There was no lymphadenopathy or heptosplenomegaly.

The full blood picture was as follows: haemoglobin concentration $7 \cdot 4 \mathrm{~g} / \mathrm{l}$; white cell count $2.1 \times 10^{9} / 1$-neutrophils $0.5 \times 10^{9} / 1$, lymphocytes $1.3 \times 10^{9} / 1$, monocytes $0.2 \times$ $10^{9} / 1$, eosinophils $0.1 \times 10^{9} / 1$; and his platelet count was $19 \times 10^{9} / 1$. Bone marrow aspirate showed fragments of variable cellularity with some dyserythropoiesis and a trepine biopsy specimen confirmed aplastic anaemia $(<20 \%$ cellularity). Repeated full viral serology, liver enzyme tests, and Ham's test were non-diagnostic. His HLA type was A2 B7 B62 Bw6 DR2 DR14.

Three months after presentation he remained transfusion dependent with a deteriorating marrow cellularity of less than $10 \%$; neutrophils were $0.3 \times 10^{\circ} / 1$ and platelets $18 \times 10^{9} / 1$ - that is, severe aplastic anaemia. The patient's mother subsequently volunteered the information that the home had been chemically treated for woodworm about two to three months before the first appearance of symptoms. Moreover, the chemical had been used on woodwork in the child's bedroom which had been hermetically sealed by polythene insulating sheets covering all the windows. The patient had reoccupied this room 48 hours after pesticide treatment. The agent was subsequently confirmed by chemical analysis from woodwork as Lindane ( $\gamma$ hexachlorocyclohexane).

\section{CASE 2}

A 28 year old male computer operator presented with a one month history of headaches, exertional dyspnoea, and easy bruising. There was no relevant medical history, recent medication, or viral infection. For several months before presentation, however, he had been actively involved in renovating an old building and had applied a proprietary solution of pentachlorophenol to timber work.

The full blood count on admission was as follows: haemoglobin concentration was $5 \cdot 3$ $\mathrm{g} / 1$; white cell count was $1.5 \times 10^{9} / 1$-neutrophils $0.5 \times 10^{9} / 1$, lymphocytes $0.7 \times 10^{9} / 1$, monocytes $0.3 \times 10^{9} / 1$; and platelet count $13 \times$ $10^{9} / 1$. Bone marrow aspirate and trephine biopsy specimen confirmed severe hypocellularity $(<10 \%)$ consistent with severe aplastic anaemia. Marrow cytogenetics were normal. A separate $36 \mathrm{mg}$ marrow biopsy specimen was frozen at $-70^{\circ} \mathrm{C}$ and subsequent analysis showed pentachlorophenol at $200 \mu \mathrm{g} /$ $\mathrm{kg}$ (control, $100 \mu \mathrm{g} / \mathrm{kg}$ ). Ham's test, full virology including hepatitis $B$ antigen, and hepatitis A IgM, yielded negative results. Biochemical profile and $B_{12} /$ folate concentrations were normal. HLA tissue type was A1 B8 Bw6 DR3 DR6.

CASE 3

A 26 year old labourer presented with a six month history of increasing fatigue and right middle lobe pneumonia. Haemoglobin concentration was $6.0 \mathrm{~g} / 1$; white cell count was $1.4 \times$ $10^{9} / 1$, with neutrophils $0.5 \times 10^{9} / 1$, lympho- 
cytes $0.7 \times 10^{9} / 1$, and monocytes $0.2 \times 10^{9} / 1$; platelet count was $10 \times 10^{9} / 1$. Autoantibody screen, full viral serology, and Ham's test yielded negative results and the biochemical profile was normal. Bone marrow aspirate and biopsy specimen confirmed severe aplasia ( $<10 \%$ cellularity). HLA tissue type was A3 A11 B5 B7 Bw4/6 DR2 DR4.

There was no relevant medical history or preceding medication. Some months before he had been occupationally exposed to Lindane while cleaning out the blocked drains from a pesticide plant at his work place.

\section{Pathology}

CASE 1

Lindane (like DDT) is a persistent residue in human adipose tissue. ${ }^{1-3} \mathrm{~A} 10.3 \mathrm{~g}$ specimen was biopsied from subcutaneous abdominal adipose tissue under general anaesthesia. Fat was extracted in Hexane $\mathrm{e}^{4}$ and the resulting solution analysed for Lindane using gas chromatography/mass spectrometry with selective ion monitoring.

Gas chromatography was performed on an OV351 capillary column with a linear temperature gradient of $120^{\circ} \mathrm{C}$ to $200^{\circ} \mathrm{C}$ over eight minutes: under these conditions the $\gamma$-isomer of Lindane eluted at seven minutes 54 seconds. Benzil (molecular weight 210), used as an internal standard, eluted at eight minutes 25 seconds.

Fragments corresponding to Lindane- $\mathrm{HCl}$ (masses 252, 254, 256) and Lindane-2 $\mathrm{HCl}$ (masses 217, 219, and 221) were clearly observed. The relative intensities of these signals and their retention times in the gas chromatography column were also characteristic of Lindane (fig 2).

The concentrations of the pure $\gamma$-isomer were about five times higher than the age matched urban control with no evidence of occupational or high exposure. The absolute value reported (25-30 parts per billion) is of uncertain importance as we could find no comparable studies using this method of analysis to establish the range of isomer concentration in the "normal" population. This result has, however, been subsequently confirmed by independent analysis.

\section{Progress}

As there was no available HLA matched sibling bone marrow donor, treatment was started with intravenous equine anti-thymocyte globulin $15 \mathrm{mg} / \mathrm{k}$ for eight days. Progress was complicated by the associated symptoms of serum sickness controlled by a reducing dose of prednisolone. Treatment was supplemented with oral oxymethalone at $2 \mathrm{mg} / \mathrm{k}$ daily. Three months later a second course of anti-thymocyte globulin was given. There was a subsequent partial response with a reticulocytosis at five weeks and an eventual increase in haemoglobulin to $11 \cdot 1 \mathrm{~g} / 1$. After 18 months without requiring transfusions he remained neutropenic and thrombocytopenic with further blood transfusions required over subsequent months.
CASE 2

Progress

Following a period of observation with no change in peripheral blood counts a central venous line was inserted and treatment started with anti-thymocyte globulin at $20 \mathrm{mg} / \mathrm{k} /$ day intravenously for eight days with oral oxymetholone $100 \mathrm{mg}$ twice a day. On completion of anti-thymocyte globulin treatment he was given (on a reducing dose) prednisolone to treat the associated serum sickness. Subsequent progress was complicated by several septicaemic episodes, which responded to intravenous antibiotics, and persistent retinal haemorrhage requiring platelet support. There was no evidence of response to anti-thymocyte globulin at 10 weeks. Although histologically incompatible in one HLA type with a sibling, the mixed lymphocyte culture was non-reactive and arrangements were made for allogeneic bone marrow transplantation. Unfortunately, he developed severe pneumonia, which did not respond to antibiotics, with sputum evidence of aspergillus. In spite of intravenous amphotericin he deteriorated with progressive pulmonary failure that required assisted ventilation. Pulmonary aspergillosis was confirmed at necropsy.

\section{CASE 3}

Progress

Initial treatment with antibiotics was started, followed by high dose intravenous methyl prednisolone at $20 \mathrm{mg} / \mathrm{k} /$ day for three days, reducing to $10 \mathrm{mg}, 5 \mathrm{mg}, 2 \mathrm{mg}$, and $1 \mathrm{mg}$ each for three days. Four weeks later peripheral blood count showed a response with haemoglobulin at $11.5 \mathrm{~g} / 1,4.7 \times 10^{9} / 1$, neutrophils 2.3 $\times 10^{9} / 1$, lymphocytes $2.0 \times 10^{9} / 1$, and monocytes $0.4 \times 10^{9} / 1$, and platelet count at $20 \times$ $10^{9} / 1$. He remained severely thrombocytopenic and progress was complicated by recurrent melaena that required blood and platelet transfusions. Continuous oral prednisolone and oxymethalone $200 \mathrm{mg}$ a day was given, but no improvement in peripheral counts was noted. Nine weeks later a course of anti-thymocyte globulin at $20 \mathrm{mg} / \mathrm{k} /$ day for eight days was administered in addition to prednisolone $30 \mathrm{mg}$ daily initially. After four weeks platelet count had increased to $44 \times 10^{9} / 1$, and over the subsequent weeks he achieved normal peripheral counts and no longer required blood and platelet transfusions. He remained well two years after initial diagnosis.

\section{Discussion}

Lindane $(\gamma$-hexachlorocyclohexane $)$ is an organochlorine that was first used as an insecticide in 1942 (fig 1). ${ }^{5}$ Its vapour pressure is low, but it is volatile enough to be used in heated dispensers. Treated wood can be guaranteed "pest free" for many years, indicating persistent biological activity. Lindane is readily absorbed transcutaneously from the lungs or gastrointestinal tract. ${ }^{6}$ The predominant body storage form in adipose tissue is the $\beta$ isomer followed by the parent $\gamma$-isomer. ${ }^{3}$ Lindane is partially metabolised in the liver to 
Figure 1 Chemical structure of Lindane shown as an analogue of myoinositol. Pentachlorophenol is a major metabolite.
Figure 2 Mass spectrometry on control (above) and patient (below) adipose tissue specimens showing intense Lindane mass 216.9145 at seven minutes 54 seconds.

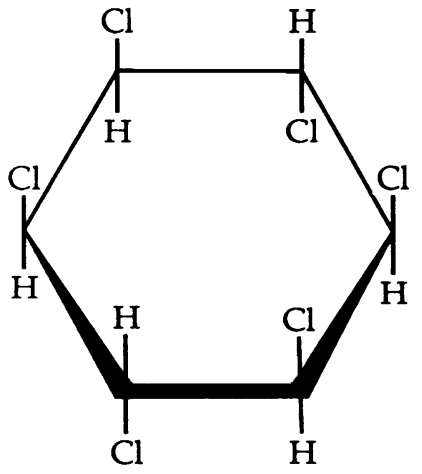

$\gamma$-Hexachlorocyclohexane

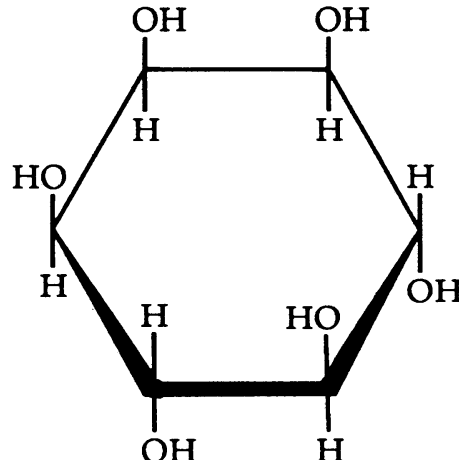

Myoinositol

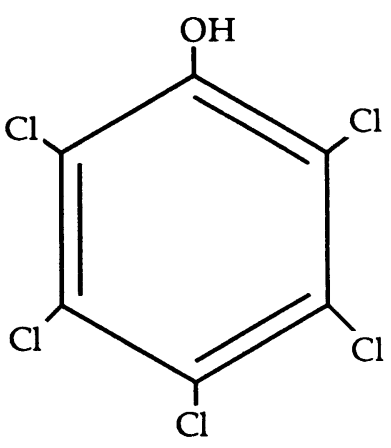

Pentachlorophenol pentachlorophenol (fig 1) (used as a wood preservative fungicide) and other cholorinated phenols. ${ }^{6}$ Acute toxicity is very well recognised as stimulation of the central nervous system and convulsions, ${ }^{7}$ particularly in children. Chronic exposure induces liver microsomal enzyme activity, and liver damage, ${ }^{8}$ including tumour formation, has been confirmed in animal studies. ${ }^{9}$ The WHO report on Lindane concluded that there was sufficient laboratory evidence that the chemical was carcinogenic in animal experiments and that it is reasonable to regard such a chemical as posing a carcinogenic risk in man. ${ }^{10}$

The association between the development of aplastic anaemia and previous exposure to drugs or industrial chemicals has been recognised for many years. This is often based on epidemiological evidence of the temporal relation between exposure and the development of aplasia, but no obvious aetiological agent is suspected in about $70 \%$ of cases. ${ }^{11}$ Clearly the importance of an accurate and detailed history of chemical exposure cannot be overemphasised.

The first nine reports of bone marrow injury associated with Lindane, particularly vapourisers, were documented in the United States by 1953 , and sharp restrictions in its domestic use were then urged. ${ }^{12}$ In spite of attempted proscription in 1971 residents in the Mid West continued to use vapourisers in their homes; by 1979 there were about 30 case reports of

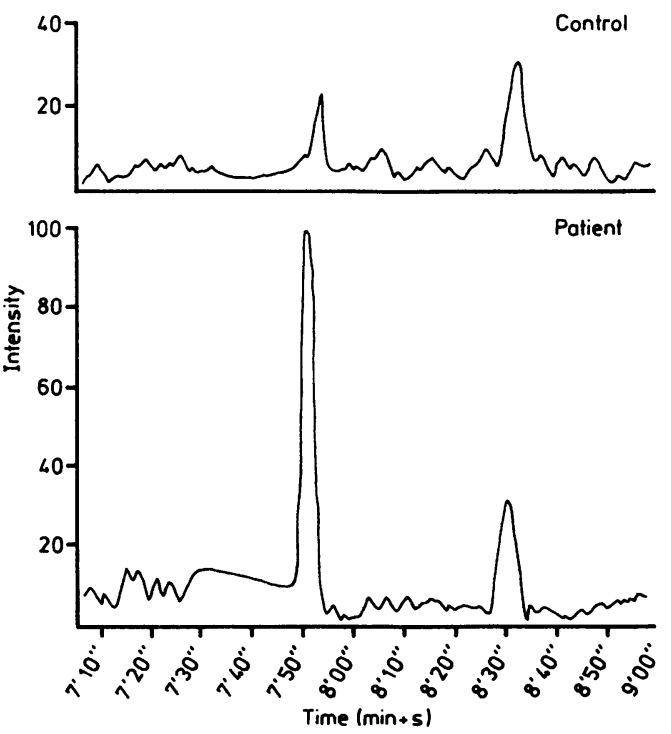

aplastic anaemia plus a further 10 cases associated with exposure to Lindane in combination with other compounds. ${ }^{13}$

Lindane has been identified in the tissues of patients in very few case reports. ${ }^{14}$ The sensitive method of mass spectrometry has only recently become available for tissue analysis and we accept that the incidence of aplastic anaemia from confirmed pesticide exposure must be very low. Lindane (hexachlorocyclohexane), however, has well documented direct biological activity: hexachlorocyclohexane is a structural analogue of inositol and inhibits phosphatidyl-inositol synthesis as the $2^{\circ}$ transmembrane messenger for growth factor stimulation. ${ }^{1516}$ Hexachlorocyclohexane or its metabolites induce chromatid breaks in vitro in human lymphocytes ${ }^{10}$ indicating potential genotoxicity to haemic stem cells.

Both the above mechanisms seem unlikely to be the cause of aplastic anaemia, as you would expect to observe inevitable dose dependent pancytopenia which is reported more commonly with organochlorine pesticide exposure. Our case reports must therefore represent a rare, probably idiosyncratic, reaction.

Aplastic anaemia has been regarded as a premalignant disorder of haemopoiesis or even a variant type of pre-leukaemia. A well documented and convincing case reports the simultaneous development of "paramyeloblastic" leukaemia in two 20 year old cousins after prolonged and simultaneous occupational exposure to hexachlorocyclohexane.${ }^{17}$ Familial or genetic sensitivity is therefore strongly suggested in these cases.

It has been shown that marrow sensitivity to chloramphenicol seems to have a genetic element and that aplastic anaemia of any cause has been associated with certain histocompatability antigens and to DR2 $2^{18}$ and $\mathrm{DPw} 3^{19}$ in particular. It may therefore be important that cases 1 and 3 both possessed DR2 antigens. Several diseases with an immunopathological component are known to be associated with the DR2 antigen, including Goodpasture's syndrome and multiple sclerosis. A cell mediated immunological mechanism has long been proposed in the aetiology of aplastic anaemia. The success of specific immunosuppressive treatment (anti-thymocyte globulin or cyclosporine) in up to half the patients with aplastic anaemia in recent trials would also support an immunological mechanism in these cases. 
Moreover, the two cases reported here which responded to anti-thymocyte globulin both shared the DR2 antigen, suggesting that there were shared features of autoimmunity.

Certain biological features associated with organochlorines and Lindane, in particular, also suggest why this agent may produce idiosyncratic marrow damage, possibly via immunological mechanisms.

Lipophilic accumulation ${ }^{1}$ Fat is an important element in the stromal support of haemopoiesis, ${ }^{20}$ and therefore a propensity of hexachlorocyclohexane to persist in marrow adipose tissue may increase the risk of interaction with lympho-haemopoietic function. For example, $\gamma$ hexachlorocyclohexane inhibits phytohaemagglutinin stimulation of lymphocyte growth. ${ }^{21}$

We suggest that organochlorine pesticides or their metabolites may become antigenic by interacting with certain rare unidentified human leucocyte antigen molecules (possibly in linkage disequilibrium with known HLA groups) and thus induce an autoimmune reaction that is responsible for the continuing marrow damage.

Apart from immunological mechanisms, idiosyncratic reactions may be due to abnormal metabolism or excretion of a chemical or drug, leading to its accumulation in excessive concentrations. One elegant study showed impaired oxidation of phenylbutazone in patients with aplastic anaemia attributed to this drug. ${ }^{22}$ Alternatively, metabolic or immunological mechanisms may be superimposed on a genetically determined haemopoietic stem cell abnormality or previous stem cell damage, thus resulting in aplastic anaemia.

Clearly there is a need for a more open and stringent evaluation of the hazards posed by agricultural chemicals. ${ }^{23}$ Little is known of the long term effects or mechanisms of toxicity of many pesticides. ${ }^{24}$ The patterns of exposure and rarity of many disorders show the limitation of purely epidemiological research. Indeed, the incidence of aplastic anaemia in developed temperate countries is only of the order of 3-6 million population per annum. ${ }^{25}$ Investigation of the aetiology of aplasia is further obfuscated by the delay of up to six months between exposure to a toxin and the development of pancytopenia. ${ }^{11}$ Well documented case reports, however, may contribute information on the suspected toxicity of these substances and serve to corroborate the recent recommendation of a standard centralised record system. ${ }^{24}$
We are grateful to Drs J Martin and PA Stevenson for permission to report their patients, and Sir Richard Body for his assistance as Chairman of the House of Commons Agriculture Committee on the effects of pesticides on human health.

The address for reporting haematological disorders and occupational hazards is as follows: Mrs J Hopkins, Haematological Disorders and Occupational Hazards Database,
Department of Haematology, University Hospital of Wales, Heath Park, Cardiff CF4 4XN.

1 Abbott DC, Goulding R. Organochlorine pesticide residue in human fat in Great Britain. Br Med J 1963;iii:146-9.

2 Baumann K, Angerer J, Heinrich R. Occupational exposure to hexachlorocyclohexane. Int Arch Occup Environ Health 1980;47:119-27.

3 Abbott DC, Goulding R, Holmes DC. Organochlorine pesticide in human fat in the United Kingdom. Hum Toxicol $1985 ; 4: 435-45$

4 de Faubert Maunder MJ, Egan H, Godly EW. Clean-up of animal fats and dairy products for the analysis of chlorinated pesticide residues. Analyst 1964;89:168-74.

5 Solomon LM, Fahrner L, West DP. Gamma benzene hexacloride toxicity-A review. Arch Dermatol 1977;113 353-7.

6 House of Commons Agricultural Committee. The effects of pesticides on human health. London: HMSO, 1987.

7 Danopoulos E, Melissinos K, Katsas G. Serious poisoning by hexachlorocyclohexane. Archives of Industrial Hygien 1953;8:582-7.

8 Herbst $M$, Weisse I, Koellmer $H$. A contribution to the question of hepatocarcinogenic effects of Lindane. question of hepatocar

9 Reuber MD. Carcinogenicity of Lindane. Environ Res 1979; 19:460-81.

10 WHO: Evaluation of carcinogenic risk of chemicals to humans: Some halogenated hydrocarbons. IARC monographs. 1979;20:220-3.

11 Gordon Smith EC. Aplastic anaemia. London: Ballière Tindail, 1989:1-15.

12 Conley BE. Health problems of vaporizing and fumigating devices for insecticides. $J A M A$ 1953;152:1232-4.

13 Morgan DP, Stockdale EM, Roberts RJ. Anaemia associated with exposure to Lindane. Arch Occup Environ Health 1980;35:307-10.

14 Loge JP. Aplastic anaemia following exposure to benezene hexachloride (Lindane). JAMA 1965;193:104-8.

15 Hoffman R, Erzbeger P. Increased phosphatidylinositol synthesis in rat embryo fibroblasts after growth stimulation and its inhibition by $\gamma$-Hexachlorocyclohexane. Biochim Biophys Acta 1980;618:282-92.

16 Parries GS, Hokin-Nearenson M. Effects of Hexachlorocyclohexane on phosphatidylinositol metabolism in mouse pancreas microsomes. Fed Proc 1984;43:1456.

17 Jedlika VL, Hermansk AZ, Smida I. Paramyeloblastic leukaemia appearing simultaneously in two blood cousins after simultaneous contact with hexachlorocyclohexane. Acta Med Scand 1958;161:447-51.

18 Chapuis $B$, Von Fleinder VE, Jeannet $M$. Increase frequency of DR2 in patients with aplastic anaemia. $\mathrm{Br}$ Haematol 1986;63:51-7.

19 Odum N, Platz P, Morling N. Increased frequency of HLA DPw3 in severe aplastic anaemia. Bone Marrow Transplant 1987;2:116.

20 Dexter TM, Whetton AD, Spooner E. The role of stroma cells and growth factors in haemopoiesis and modulation of their effects by the s.r.c. Oncogene J Cell Sci 1985;3 83-95.

21 Fisher DB, Mueller GC. Gamma hexachlorocyclohexane inhibits initiation of lymphocyte growth by phytohaemag glutinin. Biochem Pharmacol 1971;20:2515-8.

22 Cunningham JL, Leyland MJ, Delamore IW. Acetanilide oxidation in phenylbutazone associated hypoplastic anaemia. Br Med J 1974;3:313-7.

23 Coggan D. Glasnost on pesticides. Br Med J 1988;296:81-2.

24 Magos L. Thoughts on life with untested and adequately agos $L$. Thoughts on life with untested and ade

25 Szklo M, Sensenbrenner L, Marowitz J. Incidence of aplastic anemia in metropolitan Baltimore: a population based study. Blood 1985;66:115-9. 